Nature, 5 September 1996

\title{
Self-gravity as an explanation of the fractal structure of the interstellar medium
}

\author{
H.J. de Vega ${ }^{1}$, N. Sánchez ${ }^{2}$, F. Combes ${ }^{2}$ \\ ${ }^{1}$ Laboratoire de Physique Théorique et Hautes Energies, Université Paris VI, Tour 16, 1er étage, 4 Pl. \\ Jussieu 75252 Paris, France \\ 2DEMIRM, Observatoire de Paris, 61 Av. de l'Observatoire, 75014 Paris, France
}

The gas clouds of the interstellar medium have a fractal structure, the origin of which has generally been thought to lie in turbulence [1]- [2]. The energy of turbulence could come from galactic rotation at large-scale, then cascade down to be dissipated on small-scales by viscosity [3, 14]; it has been suggested that such turbulence helps to prevent massive molecular clouds from collapsing in response to their own gravity [15, 16]. Here we show that, on the contrary, self-gravity itself may be the dominant factor in making clouds fractal. We develop a field-theory approach to the structure of clouds, assuming them to be isothermal, and with only gravitational interactions; we find that the observed fractal dimension of the clouds arise naturally from this approach. Although this result does not imply that turbulence is not important, it does demonstrate that the fractal structure can be understood without it.

The interstellar medium (ISM) is an ensemble of gas clouds and dust, composed mainly of hydrogen (either atomic HI, or molecular $\mathrm{H}_{2}$ ) and helium ( $25 \%$ by mass), with other elements present in trace amounts (dust is only $1 \%$ in mass). The bulk of the ISM is distributed in cold clouds ( $\mathrm{T} \sim 5-50 \mathrm{~K}$ ), forming a very fragmented and clumpy structure, confined to the galactic plane of spiral galaxies.

For at least two decades, radioastronomy line observations ( $\mathrm{HI}$ at $21 \mathrm{~cm}$ wavelength, and $\mathrm{CO}$ at a wavelength of $2.6 \mathrm{~mm}$ for the major lines), have told us that the ISM is composed of a hierarchy of structures, with masses from about $1 \mathrm{M}_{\odot}$ to $10^{6} \mathrm{M}_{\odot}$. Structures have been observed in the ISM with sizes from $10^{-4} \mathrm{pc}\left(20 \mathrm{AU}\right.$ or $\left.310^{14} \mathrm{~cm}\right)$ to $100 \mathrm{pc}$. The largest of these structures are giant molecular clouds or complexes, thought to be the largest self-gravitating structures in the Galaxy. Above 100pc, larger structures would be destroyed by galactic shear. The accumulation of observations at many scales, and with many tracers of the ISM (CO and its isotopes, $\mathrm{HCN}, \mathrm{CS}, \mathrm{NH}_{3}, \mathrm{HI}$, dust as in Fig. 1) revealed that the interstellar medium obeys power-law relationships between size (R), mass $(\mathrm{M})$ and internal velocity dispersion $(\Delta \mathrm{v})$ (see for example [1] 3 []):

$$
M(R) \sim R^{d_{H}} \quad, \quad \Delta v \sim R^{q},
$$

These apply across the entire observed range of structure sizes and masses, with Haussdorf dimension $\left(d_{H}\right)$ and the power $q$

$$
1.5 \leq d_{H} \leq 2 . \quad, \quad 0.3 \leq q \leq 0.5 .
$$

Structures appear virialised at any scale: the scaling laws obey the relationships $\Delta \mathrm{v}^{2} \propto \mathrm{GM} / \mathrm{R}$, or equivalently $q=\left(d_{H}-1\right) / 2$.

Here we apply, for the first time, field theory and Wilson's approach to critical phenomena 伆, to the problem of a gravitational gas in statistical equilibrium. We consider a gas of non-relativistic particles in thermal equilibrium at temperature $T$ interacting with each other through Newtonian gravity. We work in the grand canonical ensemble, allowing for a variable number of particles $N$. The grand partition function of the system of particles, of mass $m$ and phase space coordinates $p$ and $q$, can be written as

$$
\mathcal{Z}=\sum_{N=0}^{\infty} \frac{z^{N}}{N !} \int \ldots \int \prod_{l=1}^{N} \frac{d^{3} p_{l} d^{3} q_{l}}{(h)^{3}} e^{-\frac{H_{N}}{k T}}, \quad \text { with } \quad H_{N}=\sum_{l=1}^{N} \frac{p_{l}^{2}}{2 m}-G m^{2} \sum_{1 \leq l<j \leq N} \frac{1}{\left|\vec{q}_{l}-\vec{q}_{j}\right|}
$$

$G, h$ and $k$ are Newton, Planck and Boltzmann constants respectively and $z$ is the fugacity $z=e^{\frac{\bar{\mu}}{k T}} ; \bar{\mu}$ is the gravito-chemical potential. Transforming this expression through a functional integral [5], 6], it can be 
shown that this system is exactly equivalent to the theory of a scalar field $\phi(\vec{x})$ (the detailed derivation will be found in de Vega, Sánchez \& Combes, 1996, in prep.); the grand canonical partition function $\mathcal{Z}$ can be expressed as a functional integral

$$
\begin{aligned}
\mathcal{Z} & =\iint \mathcal{D} \phi e^{-S[\phi]}, \quad \text { with } \quad S[\phi] \equiv \frac{1}{T_{\text {eff }}} \int d^{3} x\left[\frac{1}{2}(\nabla \phi)^{2}-\mu^{2} e^{\phi(\vec{x})}\right], \\
T_{\text {eff }} & =4 \pi \frac{G m^{2}}{k T} \quad, \quad \mu^{2}=\frac{\pi^{5 / 2}}{h^{3}} z G(2 m)^{7 / 2} \sqrt{k T}
\end{aligned}
$$

The parameter $\mu$ coincides with the inverse of the Jeans length $\mu=\sqrt{\frac{12}{\pi}} \frac{1}{d_{J}}$. The stationary points of the action $S$ are given by the equation for the undimensioned field $\phi$ :

$$
\Delta \phi=-\mu^{2} e^{\phi},
$$

which is exactly the equation satisfied by the gravitational field $U=-\frac{k T}{m} \phi$ of a perfect isothermal gas. Indeed, the equation of state combined with the hydrostatic equilibrium equation yields $\rho=\rho_{0} e^{-\frac{m}{k T} U}$. The application of the Poisson equation leads then to (5), provided that $\rho_{0}=z\left(2 \pi m k T h^{-2}\right)^{3 / 2}$. This leads to the well-known solution of the isothermal sphere, and small fluctuations around the stationary point have been studied in [7]. In terms of the scalar field $\phi$, the particle density can be expressed as $<\rho(\vec{r})>=-\frac{1}{T_{e f f}}<\Delta \phi(\vec{r})>=\frac{\mu^{2}}{T_{e f f}}<e^{\phi(\vec{r})}>$ where $<\ldots>$ means functional average over $\phi$ with statistical weight $e^{-S[\phi]}$.

The term $-\mu^{2} e^{\phi}$ which makes $S$ unbounded from below reflects the short-distance gravitational attraction. We limit the newtonian forces at short distances since there the interparticle interaction is no more purely gravitational. The ISM in isothermal conditions (i.e. the ISM in contact with the heat bath represented by the cosmic background radiation), is unstable through Jeans instability at any scale [8]; fragmentation occurs down to the scale where the coupling with the thermal bath breaks down, at which the regime becomes adiabatic instead of isothermal. This scale is that of the smallest possible fragments. Our isothermal gravitational gas model has therefore a natural cutoff here.

Now that we have derived the scalar field representing the problem of $N$-body in gravitational interaction, we work in two directions. We study first the perturbative method, then the renormalization group approach.

First we can note that $S[\phi]$ has no constant stationary points, except $\phi_{0}=-\infty$. In order to compute perturbations around $\phi_{0}$, we add a small constant term $\delta$ in the density, so that $\phi_{0}=\log \delta$ is finite for non zero values of the constant $\delta$ (de Vega, Sánchez \& Combes, 1996, in prep). The perturbative development in terms of the dimensionless coupling constant $g=\sqrt{\mu T_{\text {eff }}}$ reveals that the the field $\phi$ is massless; the two-points density correlation function decreases as $r^{-2}$ for large distances.

Therefore, the theory remains critical, for a large range of values of the physical parameters. Since we consider the gas inside a large sphere of radius $R(R \leq 100 p c$, since other forces are involved above such scale) no divergences appear at large radii. More information is gained when the perturbative development is made around the spherically symmetric solution is $\phi^{c}=\log \frac{2}{\mu^{2} r^{2}}$, which is invariant under scale transformations.

The next step to analyze this theory is to use the renormalization group. This non-perturbative approach is the most powerful framework to derive scaling behaviours in field theory (e.g. [4.9611]). As is well known, the correlation length $\xi$ for infinite volume systems becomes infinite at criticality, as $\xi \sim \Lambda^{-\nu}$ when $\Lambda \rightarrow 0 ; \Lambda=\frac{\mu^{2}}{T_{e f f}}$ is the distance to the critical point $\left(\Lambda=T-T_{c}\right.$ in condensed matter and spin systems) and varies according to the renormalization group transformations. Since here our system is critical on a finite size $R$, it is not singular and we have $\xi \sim R$, i.e. $\Lambda \sim R^{-\frac{1}{\nu}}$. The mass density $m \rho \sim e^{\phi}$ is identified with the energy density of the renormalization group (also called thermal operator). The partition function can be written as

$$
\mathcal{Z}(\Lambda)=\iint \mathcal{D} \phi e^{-S^{*}+\Lambda \int d^{3} x e^{\phi(\vec{x})}}
$$

where $S^{*}$ stands for the action at the critical point. Since the $\phi$-theory has a scaling behaviour (is critical) as seen in the perturbative approach, we can write $\log \mathcal{Z}$ as a power-law in $\Lambda$, plus an analytical function $\mathrm{F}(\Lambda)$, such that 


$$
\frac{1}{V} \log \mathcal{Z}(\Lambda)=\frac{K}{(2-\alpha)(1-\alpha)} \Lambda^{2-\alpha}+F(\Lambda)
$$

where $V \sim R^{3}$ stands for the volume, $K$ is a constant and alpha is the thermal critical exponent

Calculating the second derivative of $\log \mathcal{Z}(\Lambda)$ with respect to $\Lambda$ (at constant $V$ ) from eqs.(6) and (7) and equating the results, yields

$$
\frac{\partial^{2}}{\partial \Lambda^{2}} \log \mathcal{Z}(\Lambda) \sim K V \Lambda^{-\alpha} \sim R^{\frac{2}{\nu}}=\int d^{3} x d^{3} y[<\rho(\vec{x}) \rho(\vec{y})>-<\rho(\vec{x})><\rho(\vec{y})>]
$$

where we used that $\Lambda \sim R^{-1 / \nu}$ and the scaling relation $\alpha=2-3 \nu$ [9]. The r.h.s. of eq. (8) precisely yields the mass fluctuations squared $\left.(\Delta M(R))^{2} \equiv<M^{2}>-<M\right\rangle^{2}$. Hence,

$$
\Delta M(R) \sim R^{d_{H}} .
$$

Therefore, the scaling exponent $\nu$ can be identified with the inverse Haussdorf (fractal) dimension $d_{H}$ of the system

$$
d_{H}=\frac{1}{\nu} .
$$

On one side, the perturbative calculation yields the mean field value for $\nu$ [11]. That is,

$$
\nu=\frac{1}{2} \quad, \quad d_{H}=2 \quad \text { and } \quad q=\frac{1}{2} .
$$

On the other side, the renormalization group transformation amounts to replace the parameter $\mu^{2}$ in $S[\phi]$ by the effective one at the scale in question. This approach indicates that the long distance critical behaviour is governed by the (non-perturbative) Ising fixed point [4,9]. Very probably, there are no further fixed points [10]. The scaling exponents associated to the Ising fixed point are

$$
\nu=0.631 \quad, \quad d_{H}=1.585 \text { and } q=0.293 .
$$

From the renormalization group analysis, the two-points density correlation function behaves as $r^{\frac{2}{\nu}-6}$ or $r^{-2.830}$ for large distances $\left(r^{-2}\right.$ for mean field). This should be compared with observations. Previous attempts to derive correlation functions from observations were not entirely conclusive, because of lack of dynamical range [12], but much more extended maps of the ISM could be available soon to test our theory. In addition, we predict an independent exponent for the gravitational potential correlations $\left(\sim r^{-1-\eta}\right.$, where $\eta_{\text {Ising }}=0.037$ or $\eta_{\text {meanfield }}=0$ [9] $)$, which could be checked through gravitational lenses observations in front of quasars.

The mean field exponents describe the situation where non-linear field fluctuations are negligible. When non-linear fluctuations are strong, the renormalization group exactly accounts for their contributions, giving the Ising fixed point exponents.

If we consider the mass of the particles to be the neutral hydrogen atom, at $T \sim 3 K$, and we estimate the fugacity $z$ using the ideal gas value $z=\rho_{0}\left(\frac{h^{2}}{2 \pi m k T}\right)^{3 / 2}$, we find the length $\mu^{-1} \sim 30 \mathrm{AU}\left(4.510^{14} \mathrm{~cm}\right)$, and the dimensionless coupling $g^{2} \sim 510^{-53}$, for a density $\rho_{0} \sim 10^{10}$ atom $/ \mathrm{cm}^{3}$ ( [8] ). This extremely small $g$ supports the perturbative method at these scales implying the mean field values for the exponents (9). However, the effective coupling constant $g$ grows with the scale, according to the renormalization group flow (towards the Ising fixed point); $\mu^{-1}$ indicates the order of the smallest distance where the scaling regime applies, and corresponds to the observed smallest gravitational scale. Both numerical values for the critical exponents (9) and (10) are compatible with the values observed in the present interstellar medium eq.(2). Further theoretical work in the $\phi$-theory will determine whether the scaling behaviour is given by the mean field or by the Ising fixed point.

We have considered for the ISM the simplified view of an isothermal self-gravitating gas. This idealized view corresponds exactly to the outer parts of galaxies, far from any star formation and heating sources. There, the molecular cloud ensemble must be in isothermal equilibrium with the cosmic background radiation at T $\sim 3 \mathrm{~K}$ (e.g. 13, 8 ). Well inside the galaxy, the physics of the ISM is much more complex, especially when the violent perturbations due to star formation are taken into account. Locally, the ISM around star formation regions can effectively lose its fractal structure, at least partially (it becomes diffuse 
and much less fragmented, more or less ionized). But radiative cooling is very efficient, and shock waves are highly dissipative, so that globally on large-scale, the interstellar medium can still be considered as isothermal. The bulk of the ISM is only slightly perturbed, and we have shown that the scaling laws are stable under perturbations, so that we believe that our theory applies to most of the ISM (and in particular to the low star-forming Taurus region of figure 1).

Turbulence is probably relevant in the dynamics of the ISM [3, but this could be a consequence of the fractal structure built up by gravitational instabilities. It has been recognized for a long time that the size line-width relation in the ISM is similar to the Kolmogorov law $\Delta v \propto R^{1 / 3}$ derived for incompressible subsonic turbulence (this assumes that the energy flow per unit mass $\left(\epsilon \propto \Delta v^{3} / R\right)$ is constant all over the hierarchy, and is finally dissipated on the smallest scales through viscous processes). The energy would be powered at large scale by the galactic rotation [14]. Gravitationally driven compressible turbulence [15] as well as gravitational clouds in quasistatic virial equilibrium 16 yield mean field exponents (9).

The important point demonstrated here is that self-gravity alone can account for the fractal structure of the ISM, and quantitatively predicts its fractal dimension and related critical exponents. A new and unexpected connection between the ISM and critical phenomena uncovers. It is interesting to note that the gravitational gas has been found at critical conditions, with correlations at all scales, and scaleindependent power-law relations for a continuous range of physical parameters (temperature, coupling constant), while the spin models with which we have found an analogy, are critical only at a single value of the temperature. This feature is connected with the scale invariant character of the Newtonian force and its infinite range, i.e. $r^{-2}$.

A further step in the study of the ISM will be to include the dynamical (time dependent) description within the field theory approach presented in this paper.

FIG. 1. Image of the interstellar medium near the Taurus region at $100 \mu$ wavelength, obtained with the IRAS satellite (the continuum emission comes from dust heated by the interstellar radiation field, in this low star-forming region). The linear size of the box is 30 degrees, and the pixel is 6 arcmin. A zoom by a factor 3 in scale is made of the central region: the overall fragmented structure of the medium remains unchanged. This self-similar structure has been confirmed by the compilation of many other observations at widely different scales, revealing the fractal structure of the ISM.

[1] Larson, R.B. Mon. Not. R. Astron. Soc. 194, 809-826 (1981)

[2] Falgarone, E., Phillips, T.G., Walker, C.K. Astrophys. J. 378, 186-201 (1991).

[3] Scalo, J.M. in 'Interstellar Processes', D.J. Hollenbach and H.A. Thronson Eds., D. Reidel Pub. Co, p. 349-392 (1987) (Dordrecht, Holland)

[4] Wilson, K.G. Rev. Mod. Phys. 55, 583-600 (1983).

[5] Albeverio, S., Høegh-Krohn, R. Comm. Math. Phys. 30, 171-200 (1973).

[6] Hubbard, J. Phys. Rev. Lett., 3, 77-78 (1959).

[7] Horwitz, G., Katz, J. Astrophys. J. 222, 941-958 (1978)

[8] Pfenniger, D., Combes, F. Astron. Astrophys. 285, 94-118 (1994)

[9] Phase transitions and Critical Phenomena vol. 6, Domb C. \& Green, M.S. Academic Press, 1976 (New York)

[10] Morris, T.R. Phys. Lett. B334, 355-362 (1994).

[11] Landau, L.D., Lifchitz, E.M. Physique Statistique, 4ème édition, Mir-Ellipses, 1996 (Paris)

[12] Kleiner, S.C., Dickman, R.L. Astrophys. J. 312, 837-847 (1987)

[13] Pfenniger, D., Combes, F., Martinet, L. Astron. Astrophys. 285, 79-93 (1994)

[14] Fleck, R.C. Astrophys. J. 458, 739-741 (1996).

[15] Henriksen R.N., Turner, B.E. Astrophys. J. 287, 200-207 (1984).

[16] Chièze, J-P. Astron. Astrophys. 171, 225-232 (1987). 

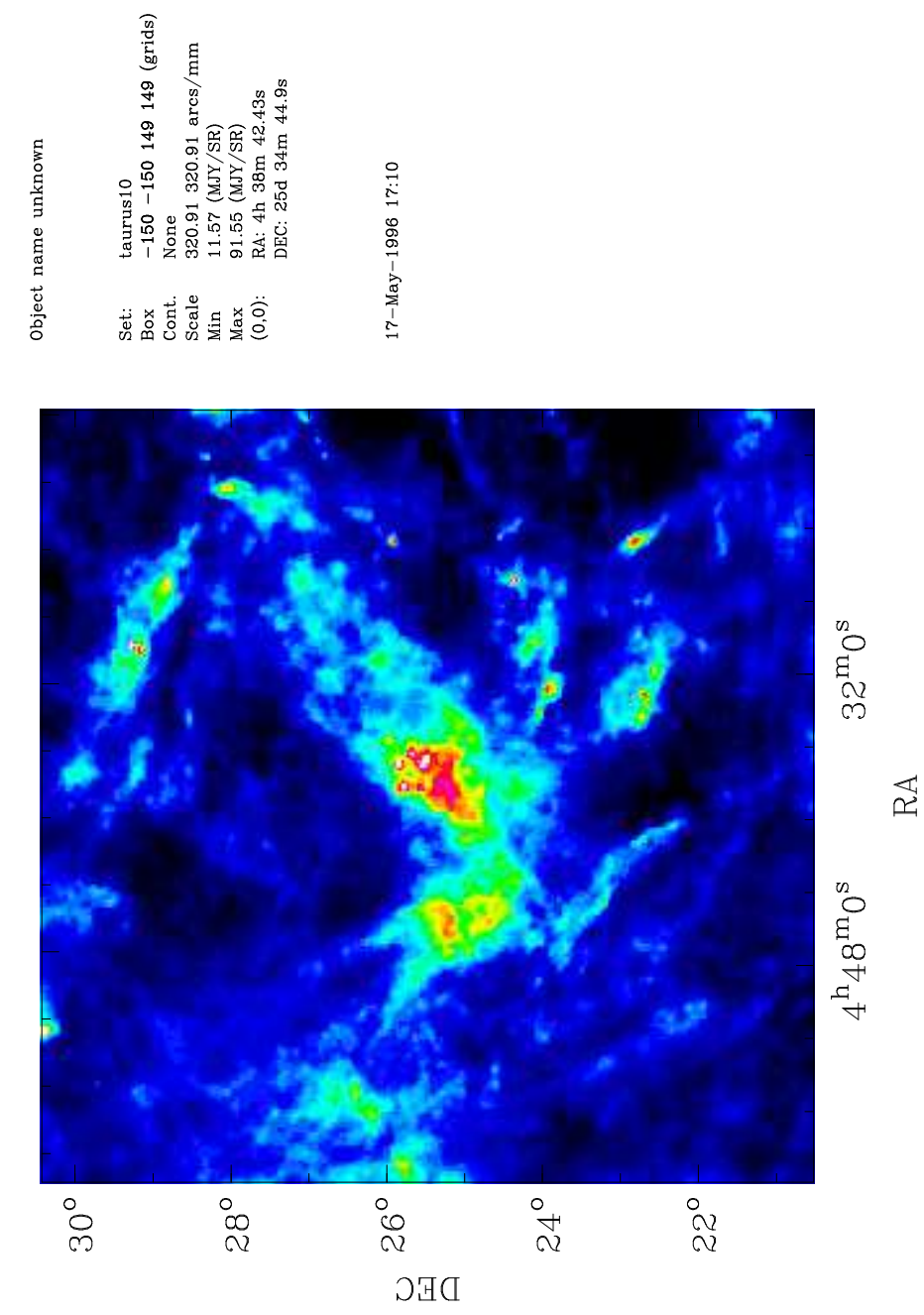


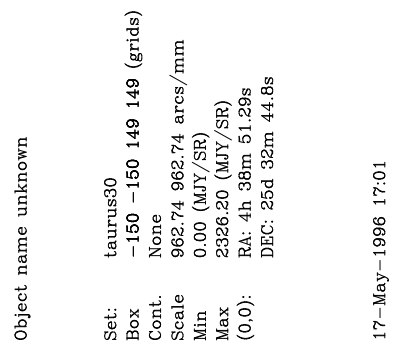

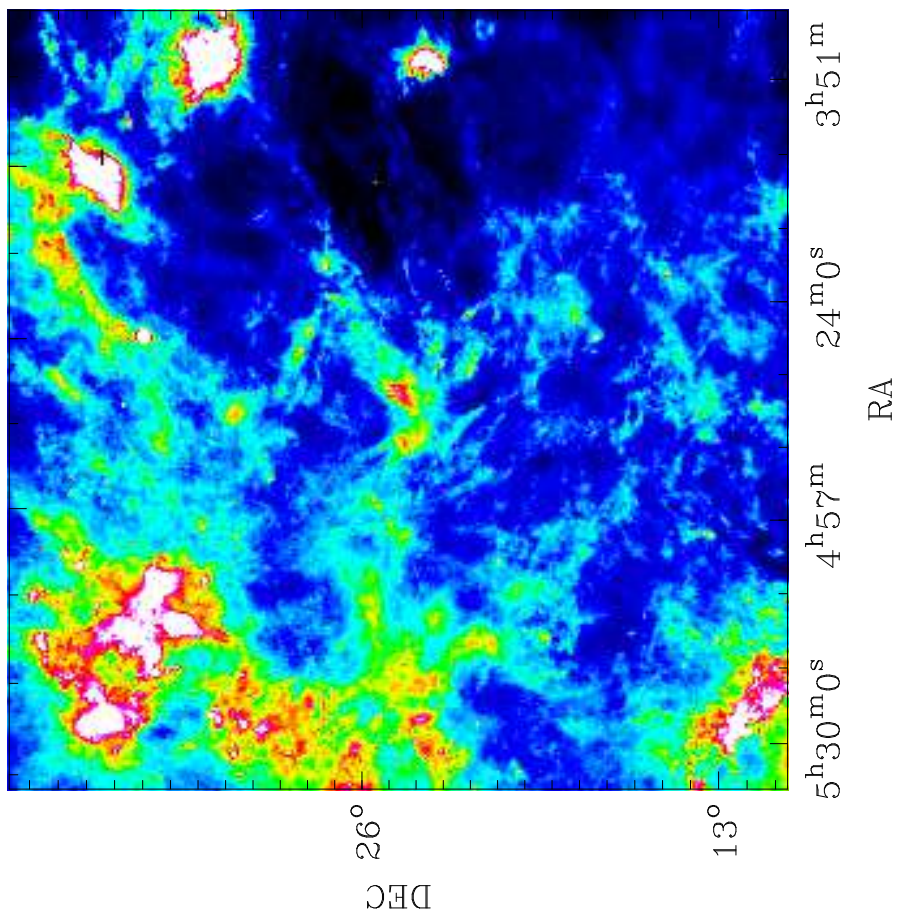

